\title{
ESTIMACIÓN DE FLUJO HEPÁTICO EN PACIENTES OBESOS SOMETIDOS A CIRUGÍA BARIÁTRICA LAPAROSCÓPICA
}

Sebastián Paredes ${ }^{1}$, Víctor Contreras ${ }^{1}$, Augusto Rolle ${ }^{1}$, Nicolás Quezada ${ }^{1}$, María Oliveros ${ }^{1}$, Luis Cortínez ${ }^{1}$

1 División de Anestesiología, Facultad de Medicina. Pontificia Universidad Católica de Chile.

Introducción: La tasa de eliminación de indocianina verde (PDR-ICG) permite estimar el flujo sanguíneo hepático (FSH) porque la ICG posee alta extracción hepática, volumen de distribución similar al plasma y no presenta eliminación extra-hepática. El monitor LiMON® permite medir PDR-ICG en plasma. No existen valores referenciales de PDR-ICG en población obesa.

Objetivo General: Describir PDR-ICG y FSH en pacientes obesos y no obesos y analizar su asociación con distintos descriptores de composición corporal.

Material y Métodos: Se incluyó 40 pacientes ASA I-II entre 18 y 60 años, sin evidencia clínica ni de laboratorio de insuficiencia hepática, operados de cirugía abdominal laparoscópica electiva. A todos los pacientes se realizó una densitometría de energía dual (DXA) previo a la cirugía para estimación de la composición corporal (masa grasa y magra). La medición de PDR se realizó en el período postoperatorio con el monitor $\mathrm{Li}$ MON® (CPulsion Medical Systems SE) luego de una dosis de $0,25 \mathrm{mg} / \mathrm{kg}$ de ICG endovenosa (máximo $25 \mathrm{mg}$ ). La volemia teórica para cada paciente se calculó mediante la fórmula descrita por Lemmens et al1: $\mathrm{EBV}=(70 / \sqrt{ }[\mathrm{IMC} / 22]) *$ peso. El FSH se calculó como: EBV* PDR/100. Para el análisis estadístico se usó test de Pearson. Se consideró significativo un valor $\mathrm{p}<0,05$.

Resultados: Se incluyeron 27 pacientes obesos (media edad 41 años, mediana IMC $36,9 \mathrm{~kg} / \mathrm{m}^{2}$ [rango 30,5 - 46,9]) y 13 pacientes no obesos (media edad 44 años, mediana IMC $25,9 \mathrm{~kg} / \mathrm{m}^{2}$ [rango 18,8 - 29,7]). No hubo asociación significativa entre el PDR-ICG y la masa magra, masa grasa, peso total o IMC (figura 1, gráfico A). El FSH estimado fue $1.384( \pm 372) \mathrm{ml} / \mathrm{min}$. El FSH se asoció en mayor medida al aumento de la masa magra $(\mathrm{r}=0,46 ; \mathrm{p}<0,01)$ que al aumento de masa grasa $(r=0,37 ; p=0,02)$ (figura 1 , gráficos B y C).

Conclusiones: El flujo sanguíneo hepático, pero no la tasa de eliminación de indocianina (PDRICG), aumenta en obesidad, correlacionándose mejor con el aumento de masa magra. Nuestros
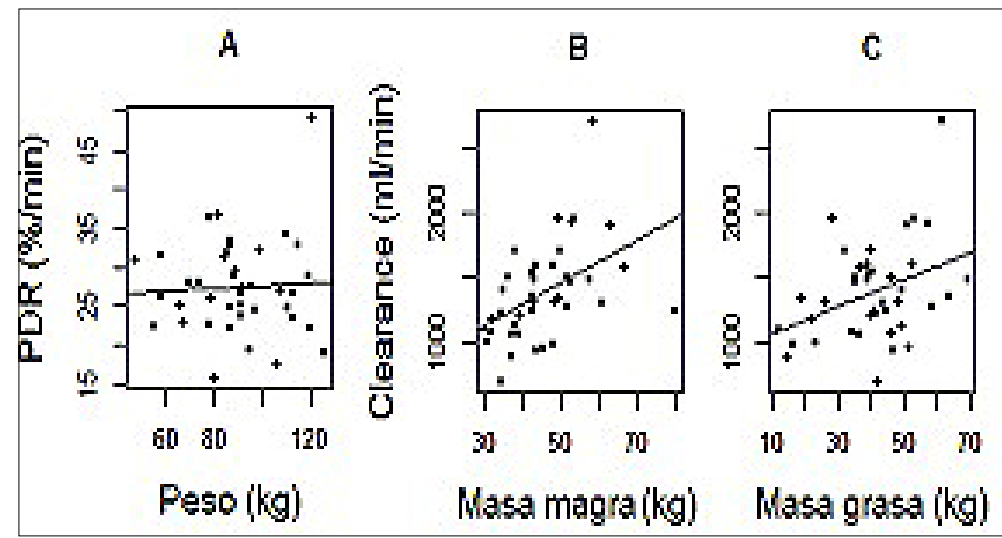

Figura 1. 
resultados sugieren que en pacientes obesos, sin evidencias de insuficiencia hepática, la eliminación de drogas de alta extracción hepática debiera ser mayor que en no obesos por el mayor flujo hepático de esta población.

\section{Referencias}

1. Lemmens HJM, Bernstein DP, Brodsky JB. Estimating blood volume in obese and morbidly obese patients. Obes Surg [Internet] 2006; 16: 773-6 Available from: http://www. ncbi.nlm.nih.gov/pubmed/16756741. 